\title{
The Choreography of Piling: Active Industry in the City
}

MARIE LAW ADAMS \& DANIEL ADAMS

Northeastern University

Gravel, salt, sand, cobbles, and scrap metal - dry bulk materials fundamental to making and maintaining the built environment - are piled in or around coastal cities. The pile is the architecture of the holding stage between a material's arrival and accumulation from one mode (such as ship or rail) and its distribution into the city through another (most commonly, the truck). Although these piles often approach the scale of large buildings and natural landforms, and their presence is a fixture in the built environment, they are overlooked as a matter of design.

In recent decades, some artists and architects have explored piles and pile-making as an abstract formal condition or alternative to conventional modes of formal organization, but engaging the pile as an active form-making structure in the city has been confined to designating territories for piles through usebased zoning protocols ("industrial"), or through the construction of containers to enclose them (sheds). Both of these standard practices fail to negotiate the distinctive qualities of piles as a temporary, kinetic, and authentic architecture in the city, and inhibit the collective engagement between the city and an expression of its global material footprint. This paper will explore the morphology of piles and present tactics for engaging them in pursuit of new notions of authenticity, monumentality, and temporality as a byproduct of global flow through three realized projects by our firm, Landing Studio, that choreograph the architecture of industrial road-salt piles in Boston and New York City.

\section{MORPHOLOGY OF ROAD SALT PILES}

Piles are constructed artifacts, made by a combination of innate physical properties and construction logics. In its most basic form, a dry pile poured from above takes the shape of a cone. The pile's angle of repose, or incline, is a result of physical properties of the material,

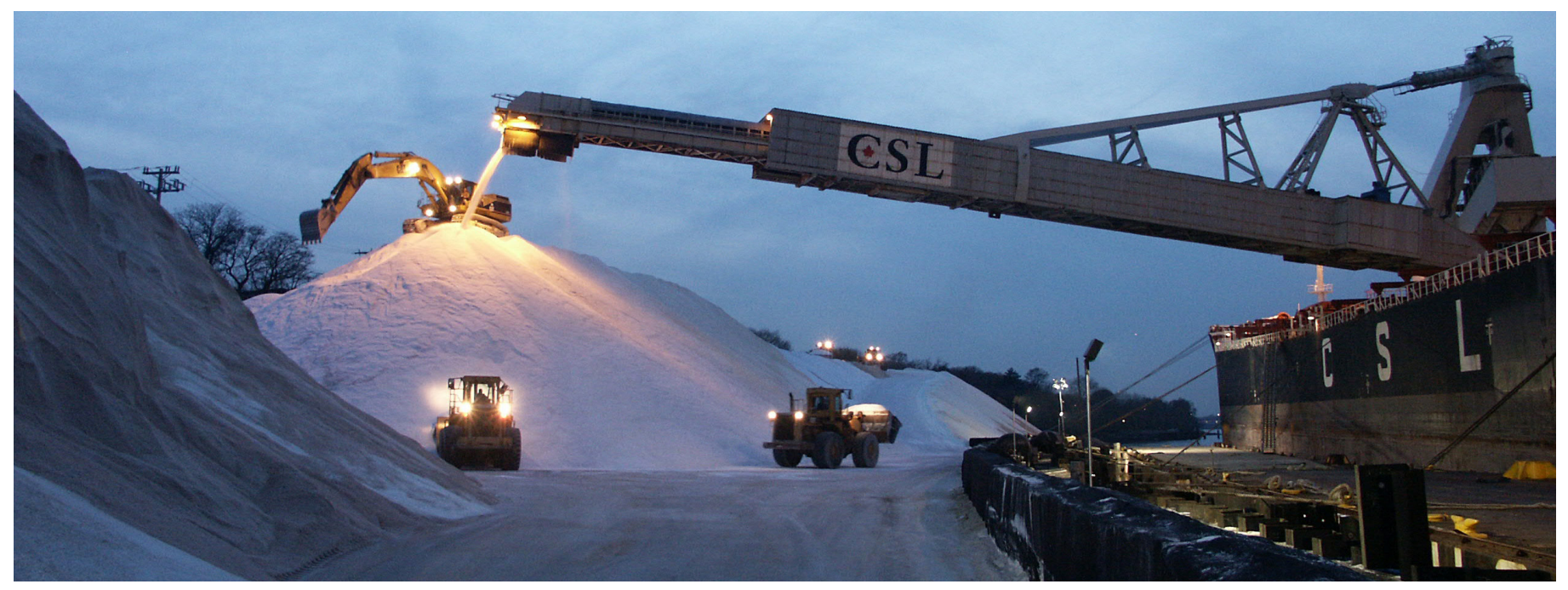

Figure 1: Self-unloader discharging salt into pile, Staten Island, NY 


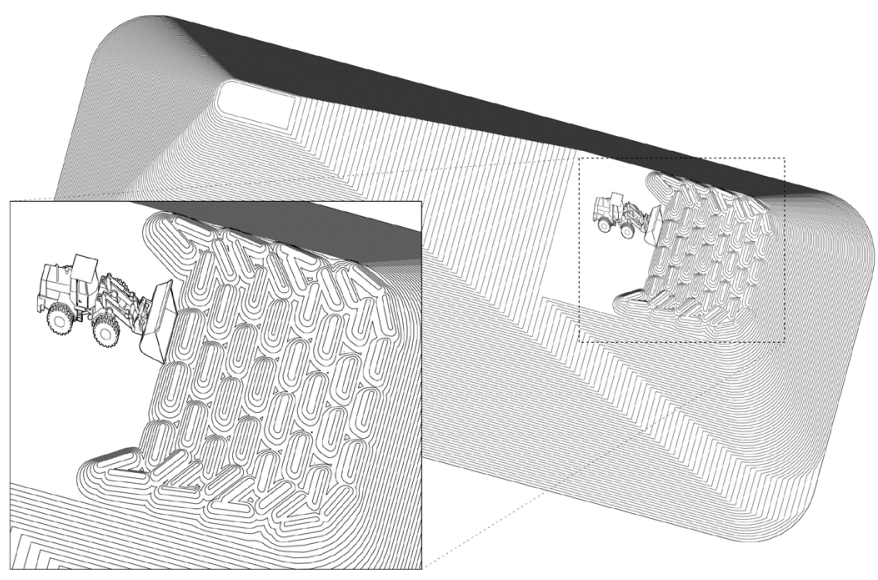

Figure 2: Front end loader deposits small piles that aggregate to larger stockpile

including the shape, size, and smoothness of its particles, such that salt, sand, and gravel piles have slightly different proportions. The pure cone form is usually seen in piles of smaller quantities, as they are the result of a construction process of dumping or pouring from above either from a conveyor or machine bucket. For dry bulk stockpiles that cover large areas, the construction process can be more complex and multi-staged, involving the use of machines that influence the final form of the stockpile.

A common method to build large piles is to use earth-moving machines to create a series of layered ramps that stack one above the other to produce a stockpile. The bottom layer and footprint of the pile is established with a series of closely spaced small piles each created by a single bucket drop or dump of material from a front end loader or truck. The small piles are then smoothed together into a thick layer with a machine bucket or blade. Each layer is constructed with an overall incline that serves as an access ramp for the machines to reach the next layer and build the pile incrementally higher. The resultant mineral mountains, or piles, are embedded with both the layered strata of this construction process and the material properties like angle of repose. For instance, a rectangular area of approximately one acre holds about 50,000 tons of salt at a height of about sixty feet, which is also the approximate carrying capacity of a Panamax dry bulk carrier.

In the case of Boston and New York City, road salt is imported from a network of extraction sites around the world. A single pile can be formed from salts of different places, extraction methods, and geologic eras, all which influence the coloration and texture of the mineral. Salt that is harvested by industrialized evaporation from a contemporary ocean or salt lake is bright white in color and soft in texture due to the crystallization process from a dissolved state. Salt that is extracted from underground or surface mines from prehistoric evaporated oceans and lakes can be a brown, beige, or pink hue, depending on the presence of other minerals accumulated over time. This salt is blasted with explosives from a solid state and broken down into smaller particles in a crushing machine, and the result is comparatively more dense and gravelly in texture. As a salt pile at a shore-side terminal is carved away after a winter storm, the layered construction is revealed through the colored and textured strata of the pile - a local deposition of global resources across space and time.

Depending on the local climate, the piles of many resource industries fluctuate with regular cycles of seasonal demand. Materials used for construction (gravel, sand, etc.) are consumed primarily in the summer, while the road salt industry is most active in the winter months. Throughout the winter season, a salt pile is constantly built up in response to ship arrivals and dismantled with weather events and local demand, creating an animated, shape-shifting environment where ordinarily static urban form changes in the course of days or even hours. In the summer months, on the other hand, salt piles typically shrink and become almost entirely stationary, temporarily opening up new open space on the urban waterfront. As such, pile landscapes are a unique physical index of dynamic seasonal, economic, and global forces in the urban environment.

\section{SALT PROJECTIONS}

The Salt Projections were a series of temporary winter-time light projections on an active road salt pile in Boston. The 150,000 ton pile is located on the shore of Chelsea Creek, in the inner harbor where the adjoining neighborhood rises uphill immediately from the industrial waterfront, creating a relationship where the salt pile during peak winter capacity forms a façade to the city. The projections responded to the kinetic characteristics of the wintertime pile, triggered by combinations of weather, local demand, and shipping schedules as the light disappeared and reappeared in accord with the industrial operations. The light took the form of very simple messages tuned to local events ("LET IT SNOW" or "VOTE") that indexed the changing pile terrain.

Light was chosen as a medium for this project because it could both engage with the substantial scale of the pile, reaching a height of sixty feet and spanning a city block, and because it could index shortterm change. The letters would become distorted as a face of the pile was carved away by a machine, or they would disappear entirely as the pile vanished after a winter storm ("SNOW" becomes "OW"). The familiarity of the projected letters and their continued legibility through deformation and disappearance enhanced one's reading of the changing pile landscape and brought the industrial operations into new dialogue with the city.

\section{LUMEN}

Lumen is a one-night video and performance art festival in Staten Island, New York, put on by an organization called Staten Island Arts that supports local art and cultural production. Every two years the summertime festival is held on a waterfront road-salt dock, and Landing Studio designs the translation of the industrial landscape into a public art venue.

Terminals for the transshipment of road salt from ocean-going ships receive 50 to 60 thousand ton cargos of material within a single Panamax ship delivery that is unloaded over the course of one to a few days, depending on the type of ship. A substantial cargo laydown area in the form of several acres of flat, paved waterfront area 

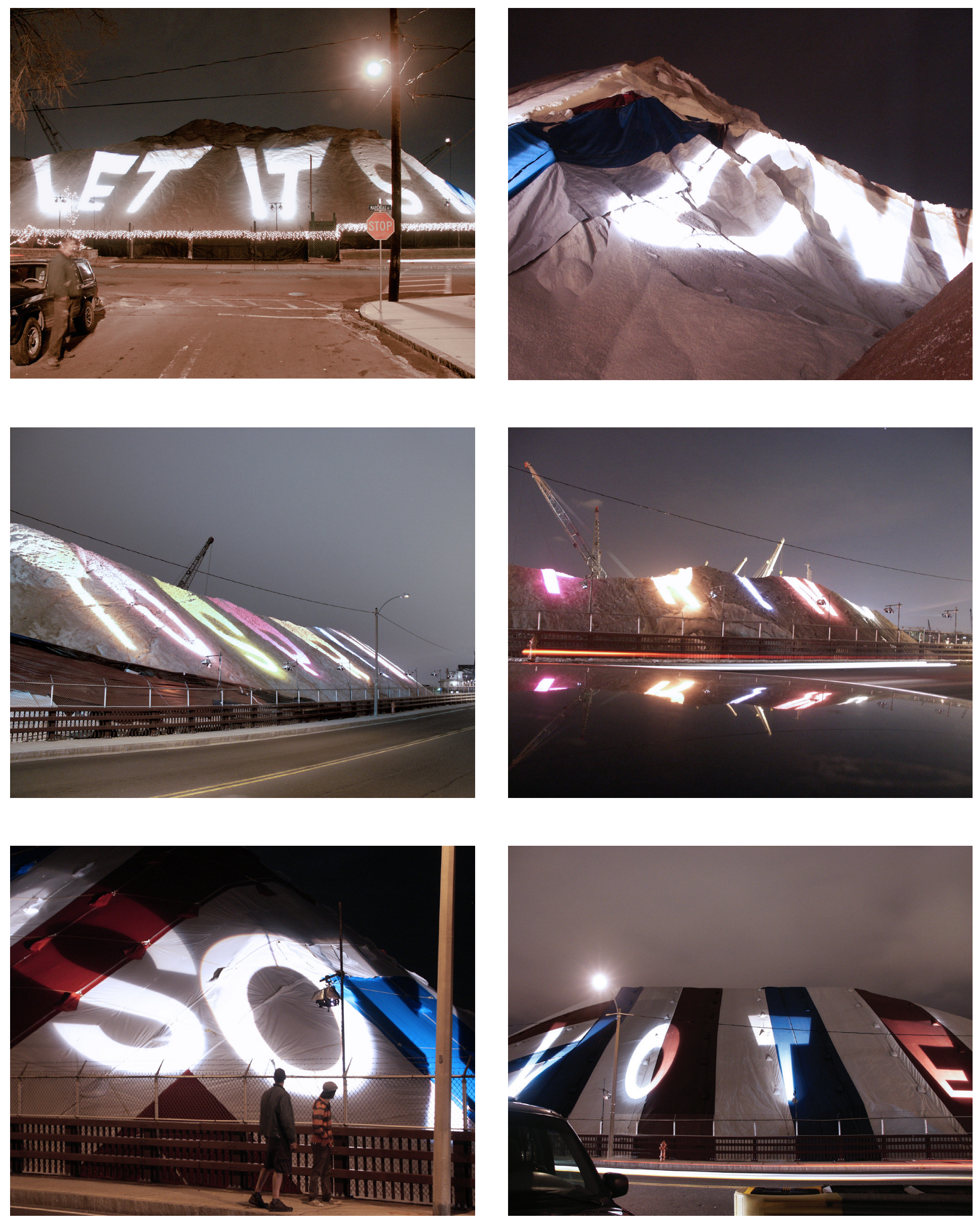

Figure 3: Light projections on the salt landscape, Chelsea, MA 


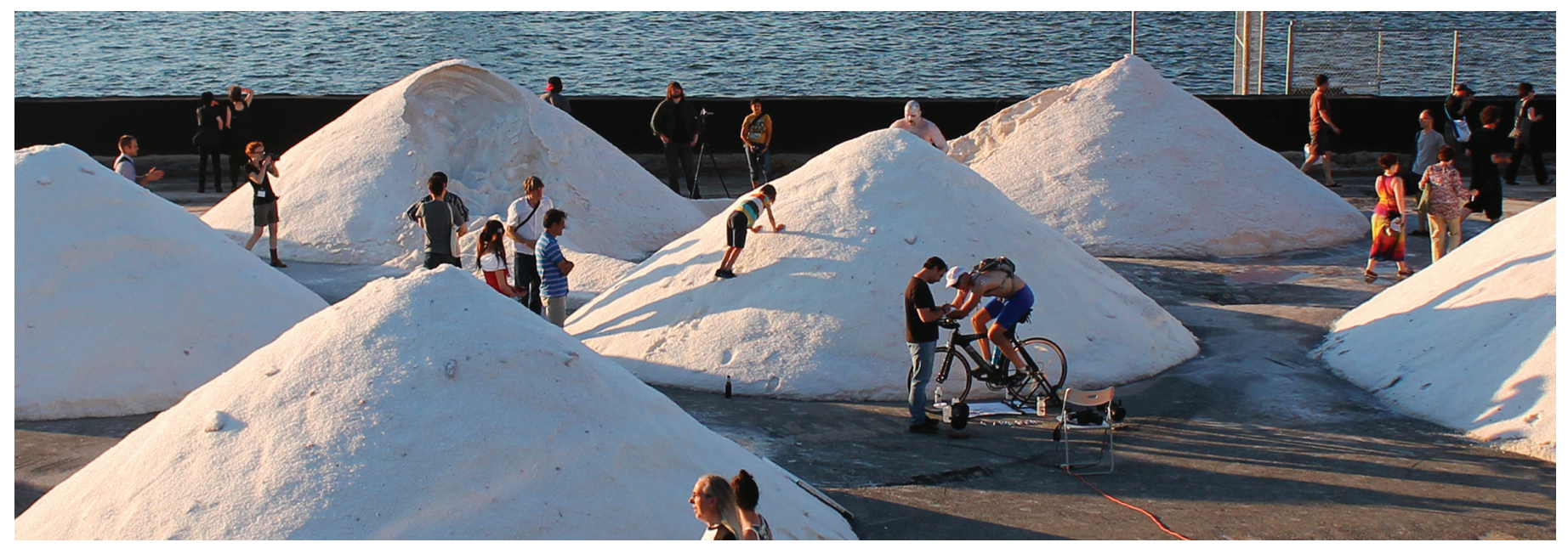

Figure 4: Salt pile landscape constructed for artists' installations, Staten Island, NY

is required to unload and stockpile such a quantity of material. So as road-salt operations slow towards the beginning of the summer and the remaining salt is consolidated in a smaller pile, a new expanse of waterfront open space surfaces on the terminal. The Lumen event takes advantage of the seasonal shift in operations and recovery of valuable open space, drawing thousands of visitors from all over the city within the course of a few hours and temporarily fills the salt stockpile area with art installations, projections, and crowds of people.

In 2012, partially owing to an unusually large salt pile resulting from a mild winter, Landing Studio designed a series of salt pile landscapes for Lumen. The pile configurations were built by the salt dock machine operators with the same equipment used in the wintertime piling operations, employing their material handling skills from industrial operations for new use. The piles were designed in two configurations. The first was an offset grid of small individual piles, each approximately 8 feet tall, 28 feet wide, and spaced 4 feet apart. The piles in the grid were of the simple conical shape naturally formed from the drop or pour of material from a machine bucket. The second configuration was an elongated pile that took the form of an ' $S$ ' shape in plan. This pile was based more closely on techniques for storing larger quantities of salt, where individual buckets of salt are smoothed together into a contiguous form. Both configurations produced a variety of spatial and figural conditions, from enclosed gallery-like rooms to linear passages, to inclined surfaces and pile peaks that offered broad views across the terminal.

The salt terrains were not pre-arranged with the fifty artists installing or performing in the event that year. Instead, they were occupied and incorporated into the work spontaneously. Some performance artists used the piles as a large scale pedestal for their living sculptures, video artists projected their work on the slope of piles, and other artists inhabited the semi-enclosed spaces of the $\mathrm{S}$ to perform their pieces. Artists even began to consume the salt as a medium within their pieces, digging and re-piling the salt into new arrangements. Visitors climbed and sat on the piles to view performances, socialize, and rest.
Landing Studio specified evaporated salt from the Pacific Ocean for the event because of its bright white color that supports light and video projection and soft texture that is inviting to handle and touch. In subsequent productions of Lumen, the salt was formally made available to the artists as a material opportunity during planning stages. Each year, more of the artists incorporate salt into the work, challenging the singular definition of the material's industrial purpose within the cultural context of the city. The project uniquely engages several dimensions of the industrial operations and translates them into a new urban experience.

\section{THE PORT}

While the Salt Projections and Lumen are temporary installations, the PORT involves a longer term designed relationship between the industrial piling operations and the city. The PORT is an expanded road-salt dock in the place of a decommissioned oil terminal in Boston Harbor. For this project, Landing Studio collaborated with the salt dock operator and attorneys to develop a series of operating protocols for the industry to more productively engage with the city.

The protocols were recorded in a legal document called the Memorandum of Agreement between the private industry and the city, comprised of scenario-based strategies to choreograph the industrial operations. For instance, as pointed out in the Salt Projections project, the salt pile is a physical interface and at times a barrier between the local neighborhood and the waterfront. In response to this condition, the Memorandum outlines a series of stockpiling configurations to store varying quantities of salt based on preserving view sheds from local public streets to the water. Larger quantities of salt left at the end of the active season are tied to a graduated fund for neighborhood improvements. While most architectural and landscape design work is confined to the duration of a project's design and construction period, the Memorandum is a vehicle that recognizes that operational characteristics of industry and expands the conventional realm of design into a living relationship.

While the stockpiling protocols engage the visual relationship of the industry to the city, the seasonality of the pile is also translated through the Memorandum into a new type of shared-use programming on the 


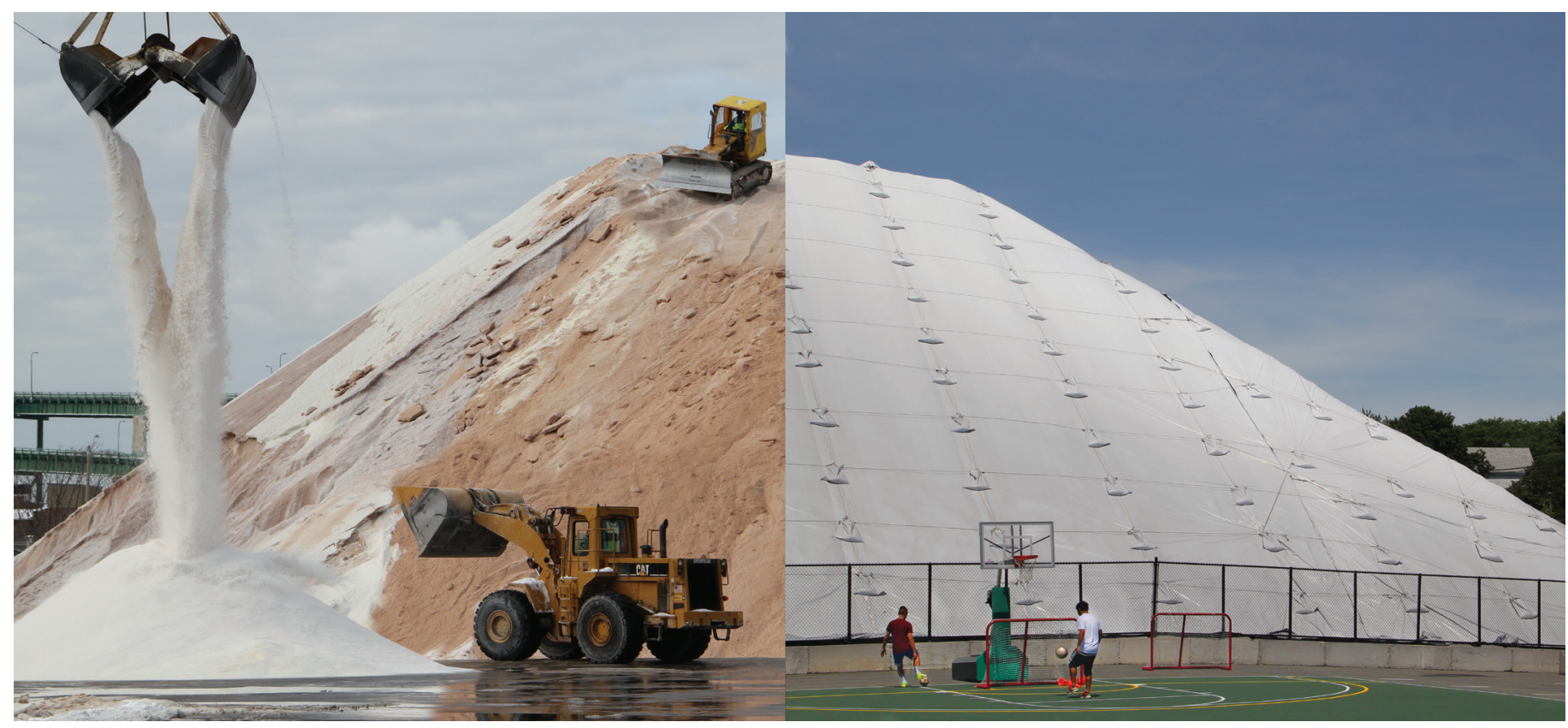

Figure 5: Winter and summer uses of the seasonally shared zone of the PORT, Chelsea, MA.

waterfront. Public waterfront access in the working waterfront zones of Boston Harbor is scarce and a source of conflict between local and regional visions for the waterfront. State and federal regulations restrict the use of large swathes of Boston's inner harbor for marine industrial use exclusively, while city zoning often directly prohibits those uses, creating a development impasse. In the PORT, the seasonal rhythm of the salt industry is translated into a solution to this challenge, where a large zone of the industrial terminal is used for salt stockpiling during the winter months and for public recreation and events during the summer. The Memorandum outlines that in the middle of May each year, the salt stockpile is pushed out of the shared-use zone to open the area for public waterfront access. As the salt is moved away, a paved ground is revealed that is striped with paint for a variety of recreational uses including basketball, a jogging track, event parking, and tent structures. Each year, in the middle of October, the salt is reintroduced in the shared zone as the industrial operations commence for the season, pairing the industrial rhythms with urban life.

\section{THE CHOREOGRAPHY OF PILES}

With their distinctive form, materiality, and animated characteristics, piles are novel yet significant physical structures in the urban environment. However, unlike traditional architecture, open spaces, or landscapes that make up the city, piles have not received much attention within the design disciplines. These three projects leverage the particular characteristics of piles and present new tactics for engaging their presence in the built environment. 